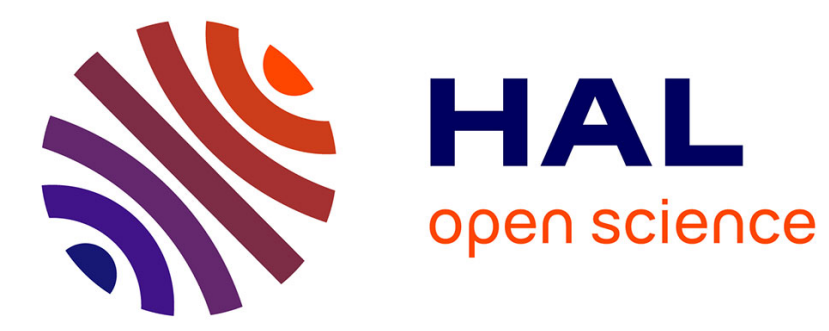

\title{
Sensitivity of the MIMO Channel Characterization to the Modeling of the Environment
}

Carlos Pereira, Yannis Pousset, Rodolphe Vauzelle, Pierre Combeau

\section{To cite this version:}

Carlos Pereira, Yannis Pousset, Rodolphe Vauzelle, Pierre Combeau. Sensitivity of the MIMO Channel Characterization to the Modeling of the Environment. IEEE Transactions on Antennas and Propagation, 2009, 57(2) (4), pp.1218-1227. 10.1109/TAP.2009.2015791 . hal-00746615

\section{HAL Id: hal-00746615 https://hal.science/hal-00746615}

Submitted on 29 Oct 2012

HAL is a multi-disciplinary open access archive for the deposit and dissemination of scientific research documents, whether they are published or not. The documents may come from teaching and research institutions in France or abroad, or from public or private research centers.
L'archive ouverte pluridisciplinaire HAL, est destinée au dépôt et à la diffusion de documents scientifiques de niveau recherche, publiés ou non, émanant des établissements d'enseignement et de recherche français ou étrangers, des laboratoires publics ou privés. 


\title{
Sensitivity of the MIMO Channel Characterization to the Modeling of the Environment
}

\author{
Pereira Carlos, Student Member, IEEE, Pousset Yannis, Vauzelle Rodolphe, and Combeau Pierre
}

\begin{abstract}
An important factor in electromagnetic wave propagation simulation, performed by a $3 D$ ray-tracing method is the modeling of the environment. Results being sensitive to descriptive accuracy, this paper presents a study on the effect of indoor environment modeling precision on multiple input multiple output (MIMO) channel characterization. In order to give some diversity to the results, the investigation takes into account two unfurnished indoor environments. The first environment investigated is the entrance hall of a building at the University of Poitiers, while the second is a more confined environment and is represented by the first floor of the authors' laboratory. For these two indoor environments, four levels of description are proposed in order to establish the geometrical and electrical modeling impact on MIMO channel characterization. Results are obtained by analyzing the capacity, variation in correlation, multipath richness and eigenvalues in relation to the polarization, the presence of line-of-sight (LOS) or non-LOS (NLOS) configurations. The effects of the spacing between antennas and the number of transmitter and receiver antennas are also investigated.
\end{abstract}

Index Terms-Capacity, correlation, environment modeling, multiple input multiple output (MIMO) channel characterization, polarization, 3D ray-tracing.

\section{INTRODUCTION}

I N MOST situations, mobile communication systems handle multimedia services in the form of wide-band systems, mobile radio channels being considered as wide-band channels. Moreover, these transmission media achieve their information propagation through a multipath mechanism. Received signals arrive from various directions, these variations being closely related to transmission quality. To overcome this drawback, traditional mobile communication systems exploit time or frequency diversity. However, to retain high spectral efficiency, one solution consists in capitalizing on spatial diversity by introducing arrays of antennas at the base and/or mobile stations of cellular networks. In order to optimize the design of array-processing algorithms, these transmissions, named multiple input multiple output (MIMO) systems, call for a thorough knowledge of the radio propagation channel. In most cases, MIMO channel characteristic parameters are evaluated by a set of measurements in

Manuscript received November 06, 2006; revised September 07, 2008. Current version published April 08, 2009.

P. Carlos is with the Signal Image and Communication (SIC) Laboratory, University of Poitiers, Poitiers, France and also with the Department of MicroWave, ENST-Bretagne, Brest, France (e-mail: pereira@ sic.sp2mi.univ-poitiers. fr).

P. Yannis and V. Rodolphe are with the Signal Image and Communication Laboratory, Department of Electrical Engineering, University of Poitiers, Poitiers, France.

C. Pierre is with the Department of Electrical Engineering, University of Poitiers, Poitiers, France.

Digital Object Identifier 10.1109/TAP.2009.2015791 studied environments, or by simulating propagation models [1], [2]. The former approach requires MIMO experimental measurement platforms, which give access to pertinent information about the radio channel for both indoor and outdoor wireless communication scenarios. However, this approach presents difficulties: for example, in relation to the calibration step and the high cost.

Consequently, an approach based on simulations is sought. There are two families of models: the statistical type and the deterministic type. The first sort is connected with experimental investigations carried out in both indoor and outdoor environments. They have the advantage of being rapid in terms of computation time. The disadvantage is that they cannot be applied to all environmental configurations. The deterministic approach is more complex, but also more accurate for MIMO characterization applications, whatever the configuration. Unfortunately, the complexity of the environment description entails that this is time-consuming in terms of computation. Considering previous research involving deterministic simulation, several studies investigated MIMO channel characterization in pico-realistic environments [3]. Variations in the spatial correlation and the capacity of the channel, based on a channel transfer matrix, were considered in relation, on the one hand, to the spacing between antennas, and on the other hand, to the number of antennas. These studies also allowed the polarization-diversity effect on MIMO channel performance to be described [2], [4]. It should be noted that the impact of complex wall structures on the capacity of MIMO wireless communication systems has been investigated [5], it being found that complex walls cannot be appropriately characterized by simple concrete slab walls. To the authors' knowledge, however, in relation to larger scale description of line-of-sight (LOS) and non-LOS (NLOS) configurations, no investigation has been carried out concerning the influence of the geometric description level and the electrical properties of the environmental materials on MIMO channel characterization. Thus, this investigation addresses the question: Is it necessary or not, for MIMO characterization, to describe the studied area in detail? More precisely, the authors set out to ascertain if an accurate MIMO channel characterization simulation is sensitive to approximations in the description of the environment, as is the case for SISO (Single Input Single Output) characterization [6].

It is to be underlined that environment modeling complexity entails inordinately large computation time. Thus, the aim of this study is also to find the simplest modeling approach that ensures a satisfactory level of accuracy while not involving excessive computation time. In this paper, we consider a deterministic model based on an optimized 3D deterministic ray-tracing 


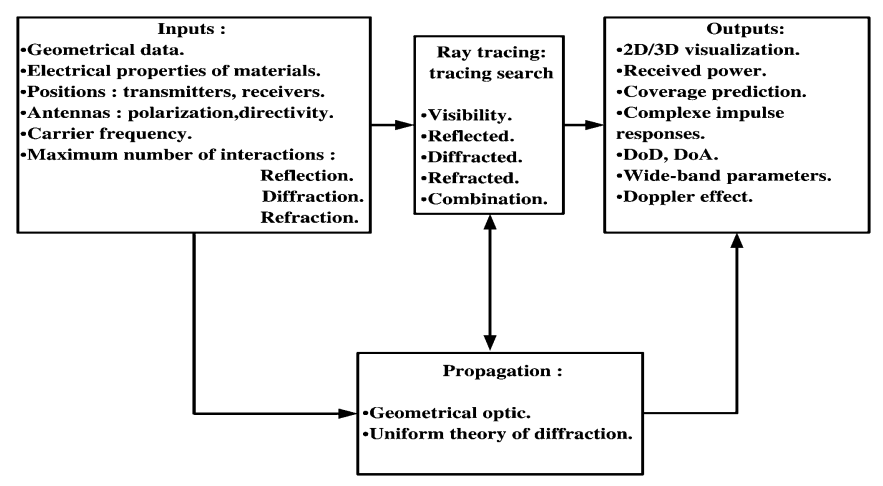

Fig. 1. Summary chart of wave propagation simulation.

method [7], [8] associated with characterization software [9]. In order to provide statistically robust results, a large number of simulations are undertaken, the approach being considered as a semi-deterministic MIMO characterization. Two indoor environments at the University of Poitiers are studied, the first being a large entrance hall of a building and the second being the first floor of the SIC ${ }^{1}$ laboratory. These environments are modeled according to four levels of description, varying from simple parallelepipeds, where all materials are assumed to be concrete, to more complex rooms taking into account the actual electrical properties of materials. Geometrical and electrical effects are analyzed by comparing channel-characteristic parameters generated at each level of description. This paper is organized as follows. Section II presents the tools and characteristic parameters of the MIMO channel (spatial correlation, capacity and eigenvalues). Section III details the indoor environment modeling. It gives the characteristics of modeling levels, including geometrical and electrical descriptions. Section IV defines simulation conditions: polarization, LOS and NLOS, the spacing between antennas and the number of antennas. Finally, Section V presents simulated results for MIMO characteristic parameters.

\section{The Modeling AND CHARACTERIZATION OF A MIMO CHANNEL}

\section{A. Modeling of a MIMO Channel}

In wireless communication, a received signal is the result of a multipath phenomenon. This is due to electromagnetic interactions (reflection, diffraction and refraction) between an electromagnetic wave and obstacles in the environment. Each path is characterized by a specific loss, polarization, propagation delay, as well as direction of arrival (DoA) and direction of departure (DoD) in azimuth and elevation [10]. The received signal derives from the sum of all the paths. To simulate this multipath mechanism, a wave propagation simulator was developed in the SIC laboratory [7]. This allows a deterministic prediction of radio channel behavior. It associates an optimized 3D raytracing technique to an asymptotic frequency method, which is based on the geometrical optic (GO) and the uniform theory of diffraction (UTD). Fig. 1 shows input and output information

\footnotetext{
${ }^{1}$ SIC: Signal Image Communications.
}

of the wave propagation simulator. The inputs concern information about the environment (geometrical and electrical properties), antennas (position, radiation pattern, polarization and carrier frequency) and electromagnetic interactions. The output information consists of the double-directional complex channel impulse response (CIR). These data are considered in order to arrive at the full characterization of the MIMO radio channel.

\section{B. Characterizing a MIMO Channel}

From the previous wave-propagation simulator output data, channel-characterization software, likewise developed in the laboratory [9], allows the calculation and visualization of characteristic functions and parameters of the SISO and MIMO channels. In this section, the MIMO channel parameters are the focus and relate to correlations between MIMO sub-radio links and the ergodic capacity which are dependent on the eigenvalues. These parameters are based on the channel transfer matrix defined in the following subsection.

1) Channel Transfer Matrix: The channel transfer matrix, $H$ is an $N_{r} * N_{t}$ narrow band matrix, $N_{r}$ and $N_{t}$ being respectively the numbers of receiver and transmitter antennas. The expression of $H$ is shown in (1)

$$
H=\left[\begin{array}{cccc}
h_{11} & h_{12} & \cdots & h_{1 N_{t}} \\
h_{21} & h_{22} & \cdots & h_{2 N_{t}} \\
\vdots & \vdots & \ddots & \vdots \\
h_{N_{r} 1} & h_{N_{r} 2} & \cdots & h_{N_{r} N_{t}}
\end{array}\right] .
$$

Each element $h_{i j}\left(i, j \in\left[1, N_{r} ; 1, N_{t}\right]\right)$ of this matrix, calculated for each radio link, is a complex narrow band coefficient. It results from the contributions of all paths making up the CIR of a given radio link $(i, j)$. Thus, $h_{i j}$ is given by (2)

$$
h_{i j}=\sum_{n=1}^{N} a_{n} e^{-j \theta_{n}}
$$

where $N$ designates the total number of paths; $a_{n}$ and $\theta_{n}$ are the magnitude and phase of the $n$th path.

2) Correlation: The first important characteristic parameter enabling the evaluation of the environmental modeling impact on MIMO propagation is the correlation [11]. More precisely, a correlation matrix of dimensions equal to $\left(N_{r} * N_{t}\right)^{2}$ is analyzed. Each element of the matrix is defined by (3)

$$
X / Y=\frac{\operatorname{cov}(X, Y)}{\sqrt{\operatorname{var}(X) \cdot \operatorname{var}(Y)}}
$$

where $X$ and $Y$ are the $h_{i j}$ of $H$, with $\operatorname{var}($.$) and \operatorname{cov}(\cdot, \cdot)$ being, respectively, the variance and covariance operators.

3) Capacity: Ergodic capacity is the second important characteristic parameter. It evaluates the performance of MIMO channels by quantifying the maximum information able to be transmitted by the propagation channel without error [12], [13]. Equations (4) and (5) or (6) and (7) define this parameter, expressed in $\mathrm{Bit} / \mathrm{s} / \mathrm{Hz}$, so long as the power of each transmitting antenna is the same

- if $N_{r} \leq N_{t}$

$$
C_{\mathrm{MIMO}}=E\left\{\log _{2}\left(\operatorname{det}\left(I_{N_{t}}+\frac{\rho}{N_{t}}\left(H H^{H}\right)\right)\right)\right\}
$$




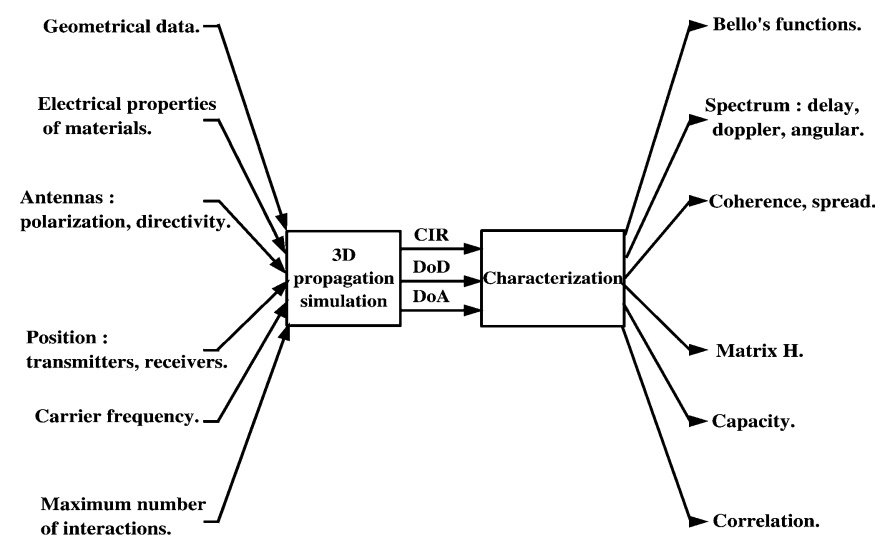

Fig. 2. Association between the wave-propagation simulator and the characterization software.

or

$$
C_{\mathrm{MIMO}}=E\left\{\sum_{i=1}^{N_{t}} \log _{2}\left(1+\frac{\rho}{N_{t}} \lambda_{i}\right)\right\}
$$

- if $N_{r}>N_{t}$

$$
\begin{aligned}
& C_{\mathrm{MIMO}}=E\left\{\log _{2}\left(\operatorname{det}\left(I_{N_{r}}+\frac{\rho}{N_{r}}\left(H^{H} H\right)\right)\right)\right\} \\
& C_{\mathrm{MIMO}}=E\left\{\sum_{i=1}^{N_{r}} \log _{2}\left(1+\frac{\rho}{N_{r}} \lambda_{i}\right)\right\}
\end{aligned}
$$

where $\lambda_{i}$ is the eigenvalues of $H H^{H}$ or $H^{H} H,(.)^{H}$ is the hermitian operator, $\rho$ is SNR (Signal Noise Ratio), $I_{N_{r}}$ and $I_{N_{t}}$ are respectively identity matrix of dimensions equal to $\left(N_{r} * N_{r}\right)$ and $\left(N_{t} * N_{t}\right)$ and $E\{$.$\} is the expectation.$

\section{Semi-Deterministic Characterization}

The evaluation of MIMO channel characteristic parameters is obtained via the association (cf. Fig. 2) of the wave-propagation simulator and the channel-characterization software. These types of software are used in order to perform a semi-deterministic study. They are thus applied to a set of a MIMO sub-radio links randomly defined. Note that this study has had to respect the stationary assumptions (WSSUS). As a consequence, the spatial variation of the antennas has been limited to small areas. Thus, the semi-deterministic study enabled by this association of software permitted the channel mean characteristic parameters (cf. Subsection II.B.2 and II.B.3) to be obtained.

\section{Modeling OF THE ENVIRONMENT}

To obtain varied results, two unfurnished indoor environments, differing in their dimensions and shape, are studied. The first one is a large entrance hall (cf. Fig. 3) which measures $28 \times 43 \mathrm{~m}$ and is made up of 4 areas. The second environment is the first floor of the SIC laboratory (cf. Fig. 4); with a surface area of $13 \times 57 \mathrm{~m}$, composed of 22 rooms. For these two environments, the impact of geometrical and electrical description on MIMO channel-characterization is investigated. In order to define this impact, four levels of description have been adopted.

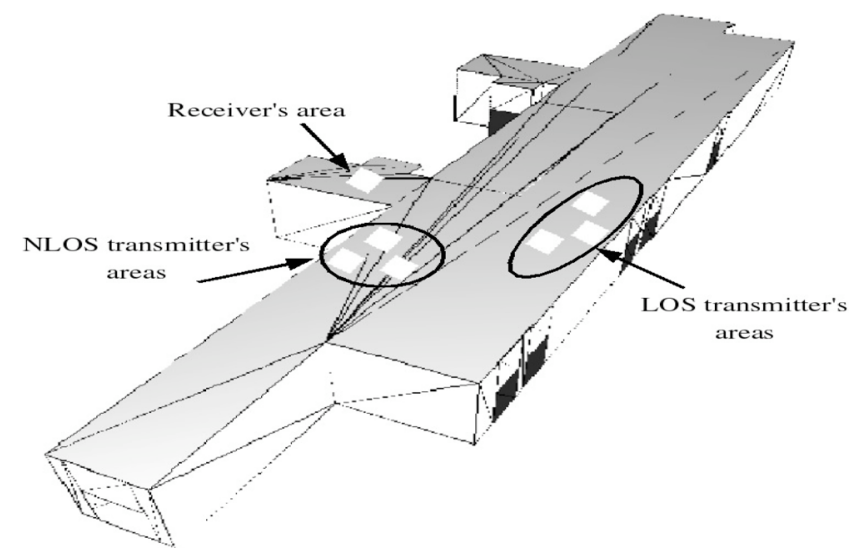

Fig. 3. Entrance hall environment.

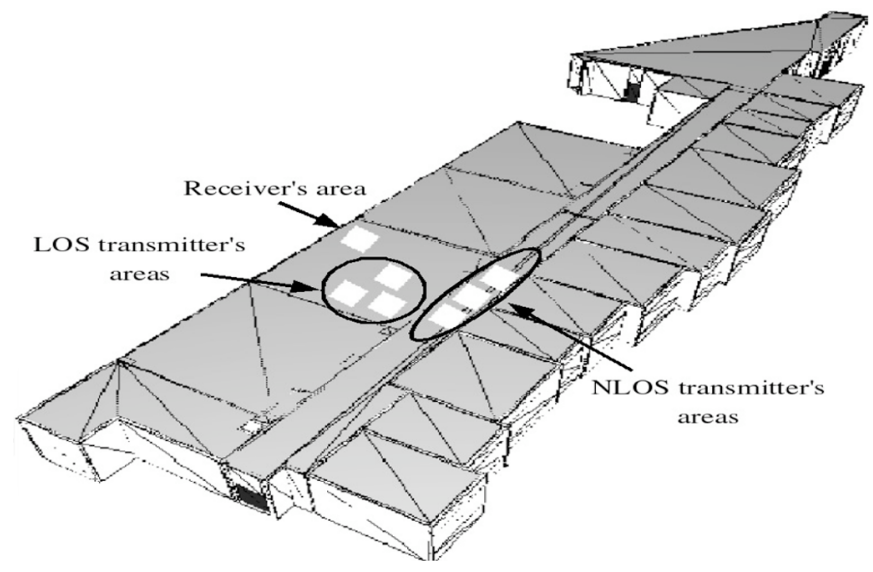

Fig. 4. First floor environment.

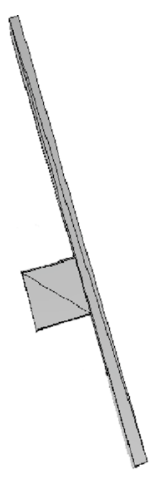

(a)

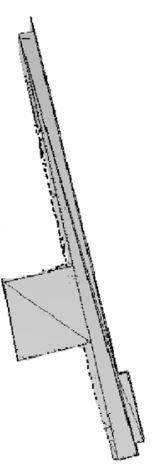

(b)

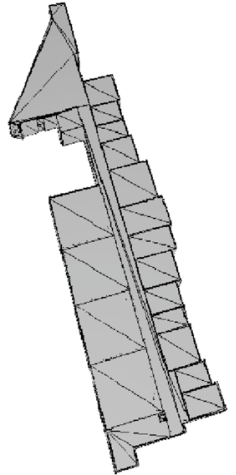

(c)

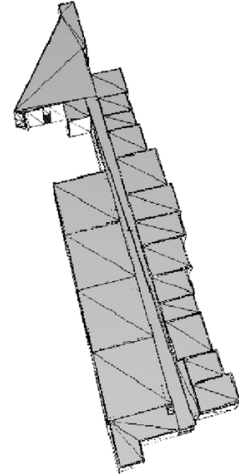

(d)
Fig. 5. Levels of description for the first floor environment. (a) Level A. (b) Level B. (c) Level C. (d) Level D.

Fig. 5 illustrates these levels of description for the first floor environment.

The first, level A is the simplest [cf. Fig. 5(a)], being described in terms of two parallelepipeds whose walls are considered to be concrete. The thickness of the interior walls is $8 \mathrm{~cm}$ and that of the exterior walls is $16 \mathrm{~cm}$. These two boxes represent the minimum level of description still allowing the investigation of LOS and NLOS configurations.

The second level [level B, shown in Fig. 5(b)] is more complex: while dealing with the same boxes as in level A, a fuller 
TABLE I

Electrical Properties of the Materials in the Studied ENVIRONMENTS, as DeduCED From Measurements [14]

\begin{tabular}{||c||c|c||}
\hline & Relative permittivity $\epsilon_{r}(\mathrm{~F} / \mathrm{m})$ & Conductivity $\sigma(\mathrm{S} / \mathrm{m})$ \\
\hline \hline Plasterboard (walls) & 6 & 0.07 \\
\hline Glass (windows) & 3 & 0 \\
\hline Wood (doors) & 5 & 0 \\
\hline Iron (radiators) & 1 & $10^{6}$ \\
\hline Concrete & 9 & 0.1 \\
\hline \hline
\end{tabular}

geometrical description is provided. Thus, to the parallelepipeds are added doors, windows and radiators according to the architectural plans. In general, doors are taken to be $200 \times 145 \mathrm{~cm}$; windows $177 \times 160 \mathrm{~cm}$ at a height of $100 \mathrm{~cm}$. Finally, radiators, $120 \times 40 \mathrm{~cm}$ in size and placed at a height of $28 \mathrm{~cm}$ are spaced at $156 \mathrm{~cm}$ intervals. They are placed only on the left-hand side of the modeled parallelepipeds. Level B retains the electrical description of level A. As a result, a comparison between levels A and B highlights properties deriving from the geometrical description.

Level C [illustrated in Fig. 5(c)] considers additional boxes besides the two of level B and employs the same geometrical precision. Thus, all the boxes making up the environment have the most accurate geometrical description possible. These additional rooms not being directly concerned by the transmission, the comparison between levels B and C shows the effect of external boxes on the characterization.

Finally, an ultimate description level, called level D [cf. Fig. 5(d)], retaining the same geometrical description as that of level $\mathrm{C}$, is considered. At this level, all geometrical elements have their correct electrical properties (taking into account: relative permittivity $\epsilon_{r}$ and conductivity $\sigma$ ), as shown in Table I. The comparison between these last two levels shows the effect of electrical properties of the environmental materials independently of their geometry. The best description was used for previous investigation [14], [15] which had validated the presented simulator with comparisons between measured and simulated results for the two environments. Consequently, the level $\mathrm{D}$ is considered as the reference of this study.

\section{Propagation Simulation Conditions}

This section presents, on the one hand, the wave-propagation simulator parameters and, on the other, the different configurations investigated.

\section{A. Wave-Propagation Simulator Parameters}

A carrier frequency of $5.18 \mathrm{GHz}$ is chosen in accordance with Wifi $802.11 \mathrm{n}$ standard. The induced wavelength is thus approximately $6 \mathrm{~cm}$. Transmitter and receiver antennas are half-wave dipoles placed at $1.5 \mathrm{~m}$ above the ground. The wave-propagation simulator, which delivers a sinusoidal waveform signal, was parameterized in such a way that a maximum of two reflections, one diffraction and five refractions were allowed for each path. In fact, these parameters had emerged from previous studies [14] of indoor SISO cases based on comparisons between simulations and actual measurements. It is helpful at this point to note that, in the current characterization, CIR are not normalized and that mutual coupling between antennas is not considered.

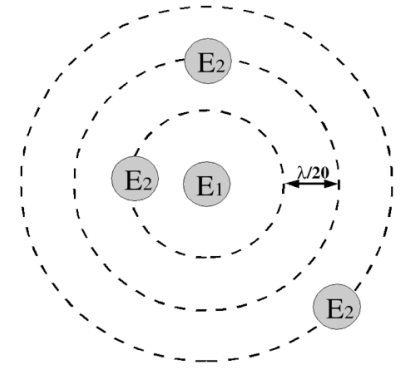

(a)

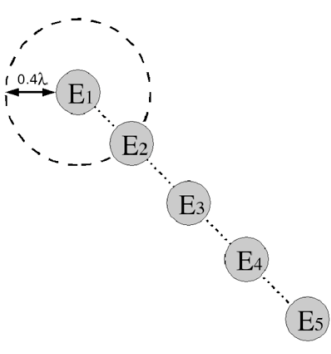

(b)
Fig. 6. Scenarios of antennas. (a) Spacing between antennas. (b) Number of antennas.

\section{B. MIMO Configurations}

In both of the studied environments, antennas vary randomly according to a uniform law in zones of one meter square. Seven zones are identified (cf. Figs. 3 and 4). One of them is dedicated to receiver antennas, the others being reserved for transmitter antennas with respect to LOS and NLOS cases. Each study takes into account three different positions. Thus, the MIMO characteristic parameters presented in Section V result from an averaged set of simulations. In the entrance hall environment, the mean radio link length is approximately $8 \mathrm{~m}$, while in the first floor environment this distance is $4 \mathrm{~m}$. The simulation is achieved with or without polarization diversity. In the first case, all antennas are vertically polarized. In the second, polarization diversity is obtained by inclining the antennas at angles of $45^{\circ}$ and $135^{\circ}$. For all the possible scenarios, the variation in the MIMO characteristic parameters is investigated in relation to:

- the spacing between antennas for a $(2 * 2)$ MIMO transmission. In transmitter and receiver areas, two antennas are in the first place randomly positioned at a distance of $(\lambda / 20)$. Afterwards, the spacing is augmented in a stepwise fashion, each spatial step being $(\lambda / 20)$ [cf. Fig. 6(a)].

- the number of antennas. The number of transmitter and receiver antennas grows from one $((1 * 1)$ SISO) to seven $((7 * 7) \mathrm{MIMO})$. The first two antennas are randomly positioned and the others follow the alignment of these two. The result is a linear antenna array, the spacing between antennas being fixed at $0.4 \lambda$ [cf. Fig. 6(b)]. This generally corresponds to a minimum correlation [16], although this value directly depends on what environments and scenarios are considered, as well as the type of antenna used.

For each possible combination of parameters, involving the visibility, the spacing between antennas, the number of antennas and the polarization diversity, 7000 simulations were carried out for a particular environment. Overall, this study represents 112000 simulations. In the rest of this work, the gain in capacity 


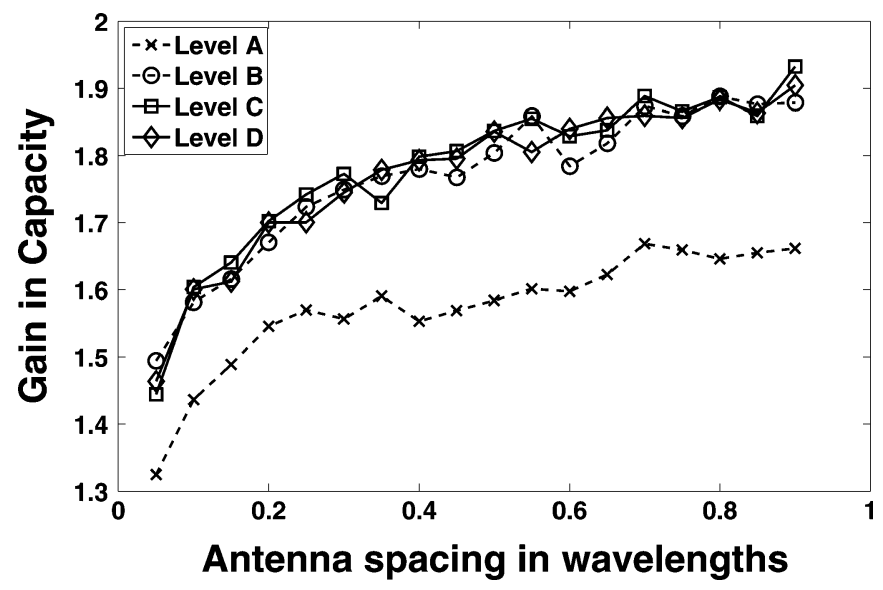

Fig. 7. Entrance hall environment: variation in the gain in capacity in relation to the spacing between antennas in LOS, without polarization diversity.

$G_{c}$ is addressed. Defined in [5], this gain results from the normalization of the average capacities by considering the average capacities of the single transmission, single receiver antenna system (i.e., $N r=N t=1$ ). Moreover, these equivalent SISO capacities are obtained for the highest level of description (level D) and for the considered polarization. Consideration of $G_{C}$ thus frees the investigation from needing to take account of transmission and reception constraints. These constraints include normalization problems related to CIR, transmission power, reception amplification and SNR. This last-mentioned parameter is kept at $30 \mathrm{~dB}$ throughout the study.

$$
G_{c}=\frac{C_{\mathrm{MIMO}}}{C_{\mathrm{SISO}}} .
$$

\section{RESUlTS}

\section{A. Variation in Characteristic Parameters in Relation to the Spacing Between Antennas}

In this section we present simulation results for the variation in the characteristic parameters (gain in capacity, correlation matrix, multipath richness and eigenvalues) with respect to the spacing between antennas.

1) Los Configuration Without Polarization Diversity: In this subsection, transmitter and receiver antennas are in LOS and do not involve polarization diversity. This means that CIR are composed of a set of paths, including the direct path, which is the most powerful. Figs. 7 and 8 show the increased gain in capacity and highlight this in relation to the spacing between antennas. Whatever the level, this gain increases up to approximately $0.4 \lambda$ [16]. Fig. 8 shows that the level of description does not affect the evaluation of gain in capacity. For the entrance hall environment, however, there is a great difference between level A and the other three levels. Thus, an oversimplified geometrical description cannot allow a pertinent evaluation of channel characteristic parameters.

To complete these observations, the focus firstly shifts to the correlation between MIMO sub-radio links. Next to be addressed are the cumulative functions associated with multipath

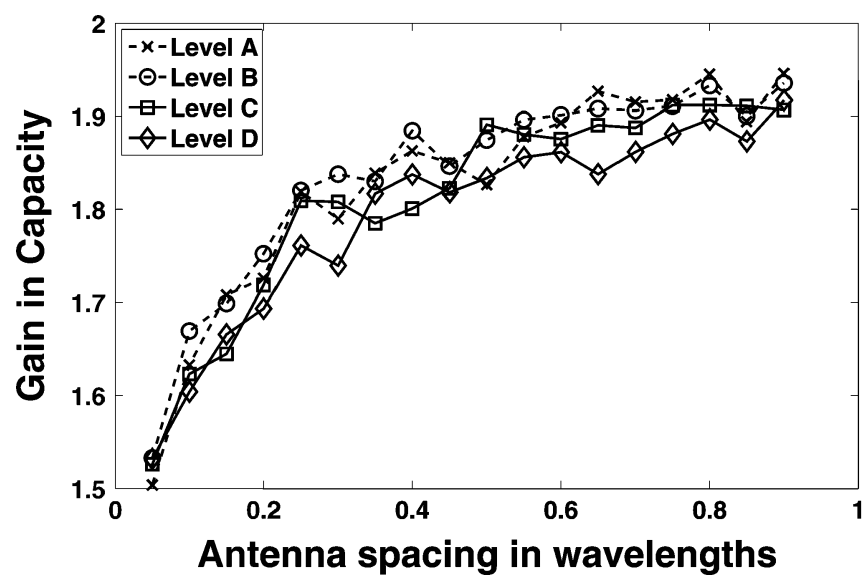

Fig. 8. First floor environment: variation in the gain in capacity in relation to the spacing between antennas in LOS, without polarization diversity.

richness. Consideration is also given to the eigenvalues of the $\mathrm{H}$ non-normalized matrix of the channel for all the studied MIMO radio links. These characteristic parameters are evaluated in the same environments under the same conditions.

In the correlation matrices only the inter-correlations between the different MIMO sub-radio links have been analyzed. The curves shown in Fig. 9(a) and (b) emerge:

- curves $h_{11} / h_{21}$ and $h_{12} / h_{22}$ involve CIR emanating from the same transmitter;

- curves $h_{11} / h_{12}$ and $h_{21} / h_{22}$ involve CIR arriving at the same receiver;

- curves $h_{12} / h_{21}$ and $h_{11} / h_{22}$ involve CIR coming from both transmitters and going to both receivers.

The variation in the correlations is similar to that of a Bessel function, with minimum values at $0.4 \lambda$ and $0.8 \lambda$, in line with previous studies using dipolar antennas [16].

As shown in Fig. 9(b), correlations vary in a similar way with respect to description levels. This similarity is supported by the results relating to capacity. However, for the entrance hall environment [cf. Fig. 9(a)], inter-correlation characteristic variation at level $\mathrm{A}$ is different from that found at the other levels. This means that geometrical description (in this configuration) has an impact on channel-characteristic parameters. This difference can be noted in curves $h_{11} / h_{21}$ and $h_{12} / h_{22}$. Examining these curves suggests that MIMO characterization depends on a minimum of geometric description of rooms that is directly related to the transmission, but seems to be insensitive to an electrical description (cf. comparison between levels $\mathrm{C}$ and D).

The previous analysis is reinforced by the curves shown in Figs. 10, 11, 12, and 13, corresponding respectively to the cumulative functions of multipath richness and eigenvalues of the simulated MIMO radio links in the entrance hall and the first floor environments.

In general, it is observed that the large number of multipaths rises from level A to level D, in line with the increasing descriptive precision of the two environments considered. This was the case irrespective of the scenarios investigated. Furthermore, the average eigenvalues associated with level A are lower than those for the other levels for the entrance hall environment $\left(6 \times 10^{-8}\right.$ at $50 \%$ for level $\mathrm{A}$, versus approximately $4 \times 10^{-7}$ 

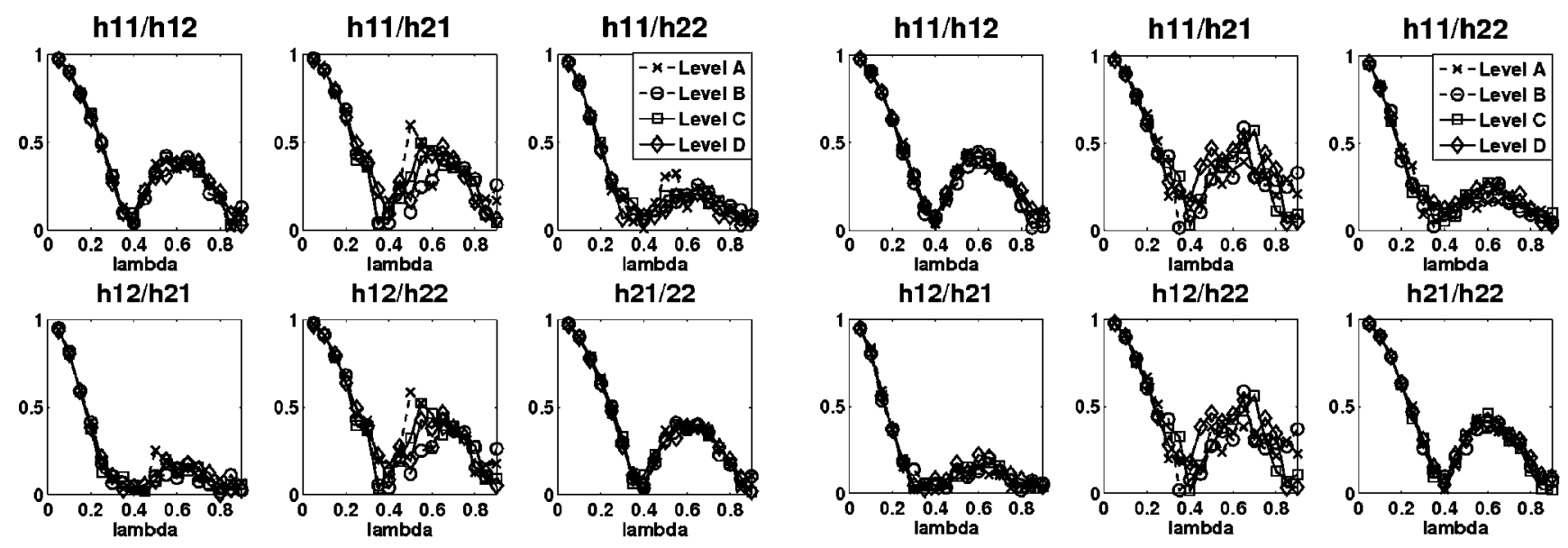

(a)
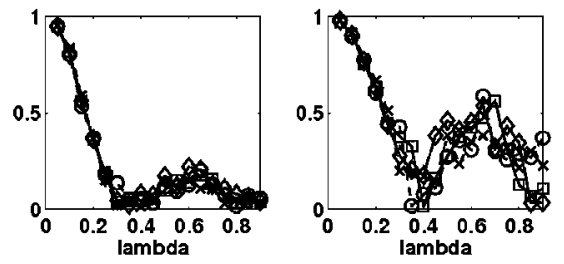

(b)

Fig. 9. Variation in correlation in relation to the spacing between antennas in LOS, without polarization diversity. (a) Entrance hall, (b) First floor.

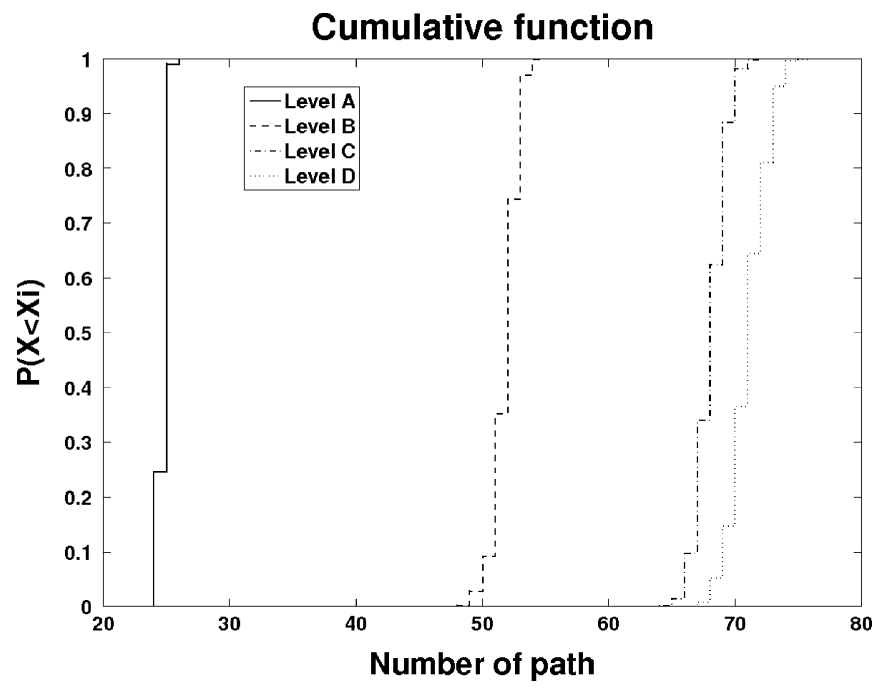

Fig. 10. Entrance hall environment: cumulative functions associated with the multipath richness in a LOS configuration, without polarization diversity.

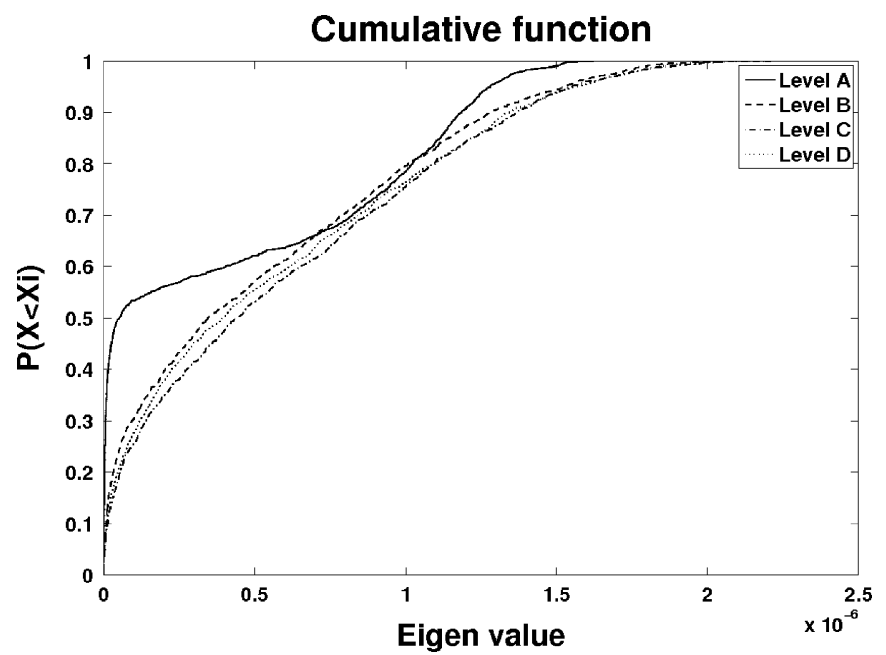

Fig. 11. Entrance hall environment: cumulative functions associated with the eigenvalues in a LOS configuration, without polarization diversity.

for the other levels). This leads to a smaller spread of the eigenvalues for level A and to a weaker capacity. For the first floor

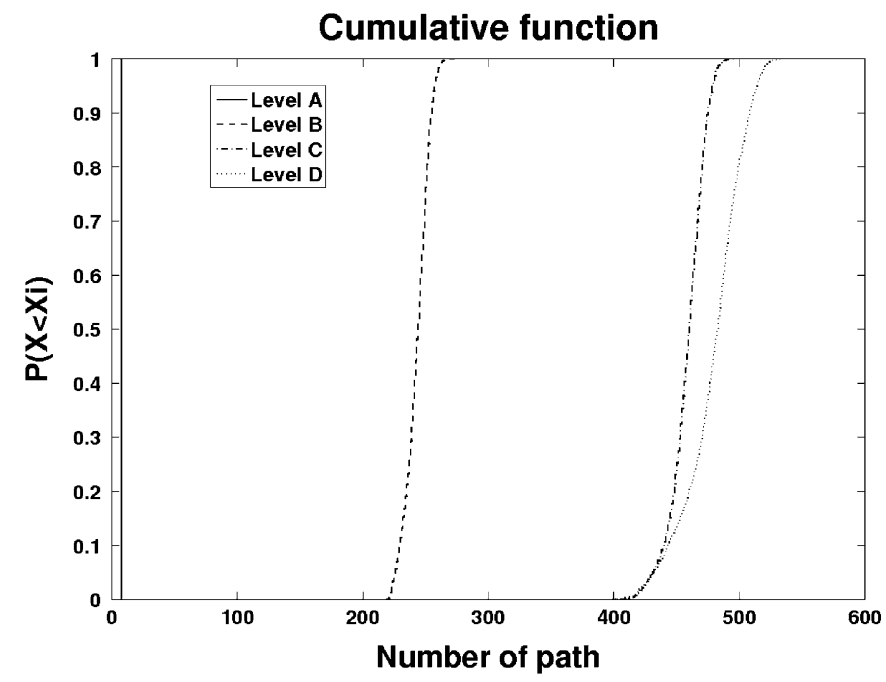

Fig. 12. First floor environment: cumulative functions associated with the multipath richness in a LOS configuration, without polarization diversity.

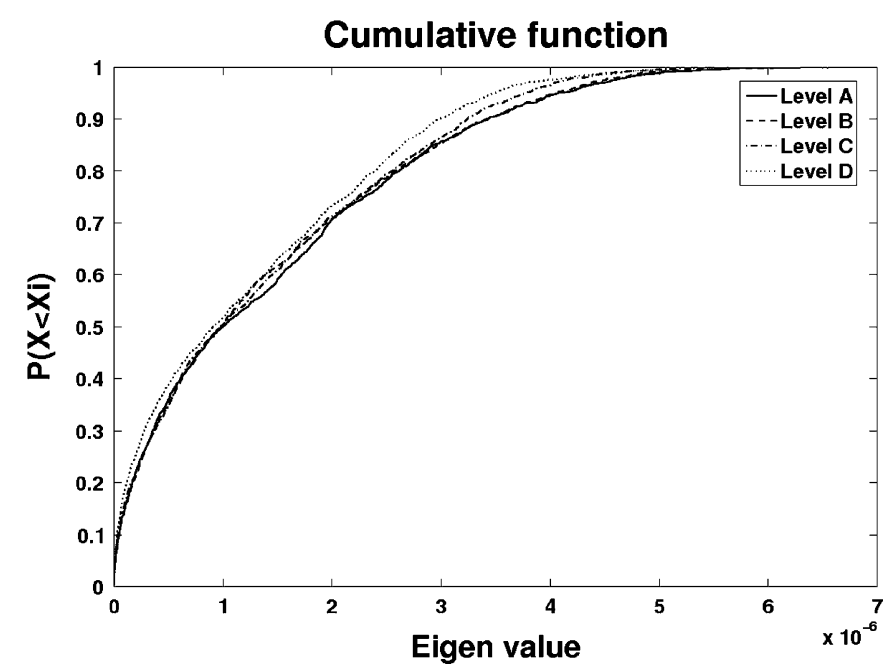

Fig. 13. First floor environment: cumulative functions associated with the eigenvalues in a LOS configuration, without polarization diversity.

environment, the eigenvalues resemble each other for all four levels (approximately $1 \times 10^{-6}$ at $50 \%$, whatever the level). This 


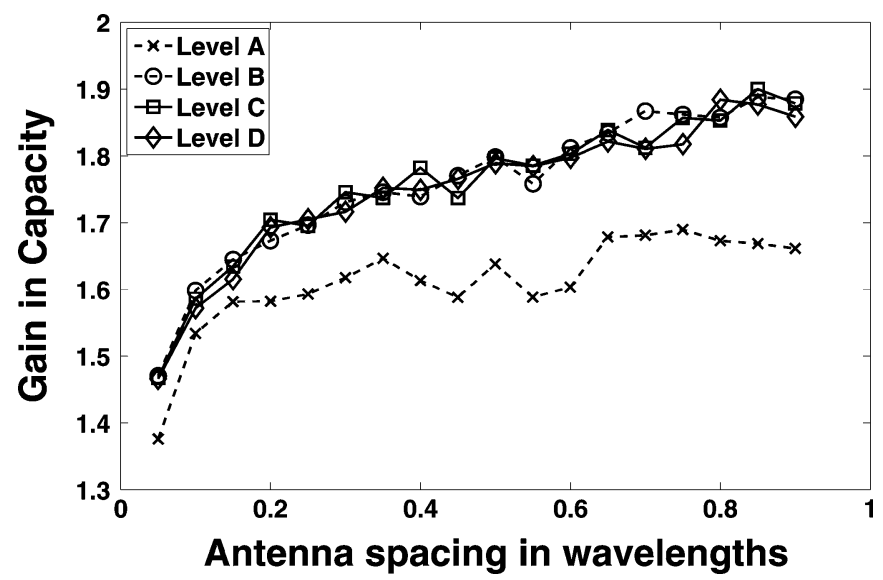

Fig. 14. Entrance hall environment: variation in gain in capacity in relation to spacing between antennas in LOS, with polarization diversity.

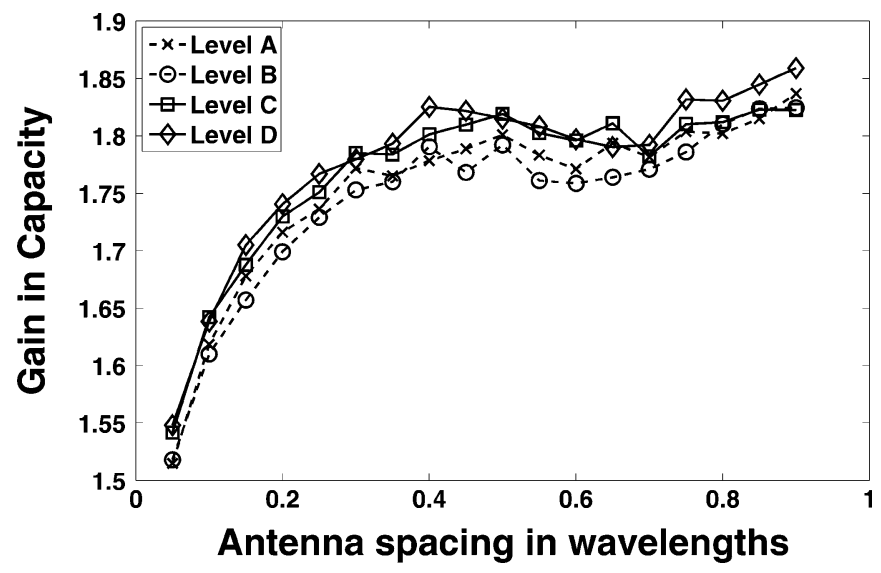

Fig. 15. First floor environment: variation in gain in capacity in relation to the spacing between antennas in LOS, with polarization diversity.

leads to changes in capacity close to the level in the other environment. These features were observed for all other scenarios, showing that in the majority of the cases, only the distribution of the eigenvalues associated with level A differs significantly from those of the other levels.

2) LOS Configuration With Polarization Diversity: Picking up on the above results, an attempt is made to determine whether or not polarization diversity increases the effects of environment modeling on MIMO characterization. Figs. 14 and 15 show that the pattern of gains in capacity corroborates the findings relating to gains in capacity without polarization diversity [cf. Figs. 7 and 8]. Accordingly, comparison between the results in Subsections V.A.1 and V.A.2 indicate that the impact of environment modeling on MIMO characterization is the same, whatever the antenna polarization in a LOS configuration.

It can be noted that the trend in the results shown in Subsections V.A.1 and V.A.2 is similar. This is due to the definition of gain in capacity (normalization of MIMO capacity by SISO capacity) being specified without recourse to units of measurement.

3) NLOS Configuration Without Polarization Diversity: The main difference between the LOS and NLOS configurations is that, in the latter, there is no longer a direct path. A focus on this condition allows the influence of the direct path on environment

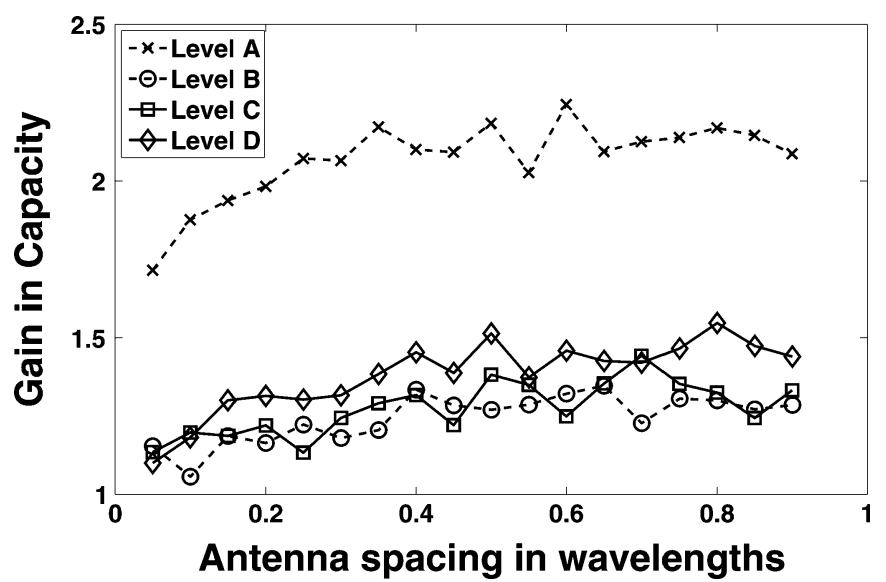

Fig. 16. Entrance hall environment: variation in the gain in capacity in relation to the spacing between antennas in a NLOS, without polarization diversity.

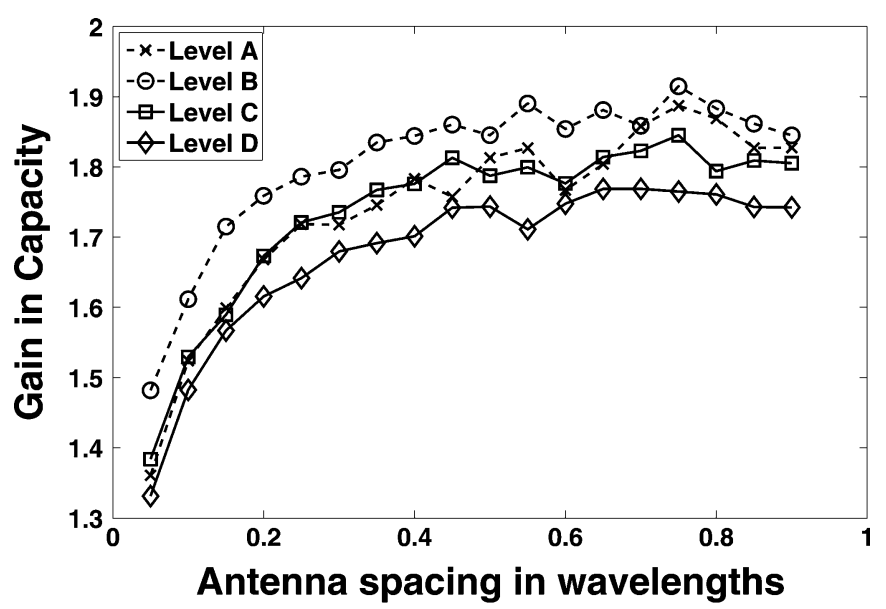

Fig. 17. First floor environment: variation in the gain in capacity in relation to the spacing between antennas in a NLOS, without polarization diversity.

modeling to emerge. The comparison between, on the one hand, Figs. 16 and 17 and, on the other, Figs. 7 and 8 points to similar characteristic trends. Nevertheless, in the NLOS configuration, the difference between the simplest geometrical description (level A) and the others is greater than in the LOS condition.

We observe, however, for the entrance hall environment that the change in capacity associated with level $\mathrm{A}$ is greater that for the other levels. This can be explained by the curve in Fig. 18 showing that the eigenvalues of level A give an average $(1.2 \times$ $10^{-8}$ at $50 \%$ for the level $\mathrm{A}$, versus approximately $7 \times 10^{-9}$ for the other levels) and a dispersal more substantial than those for the other levels. This means not only that level A generates an insufficient number of rays, but also that the rays generated have incorrect amplitudes.

Finally, it should be noted that these analyses confirm those in the literature [17]-[19] showing that the power of the rays has a more important impact on the performance of the MIMO channel than impact associated with the multipath richness.

However, characteristic parameters seem again to be insensitive to electrical description and additional architectural elements. This demonstrates that a direct path modifies the effects of environment modeling. Supporting this, the same results are obtained with polarization diversity. 


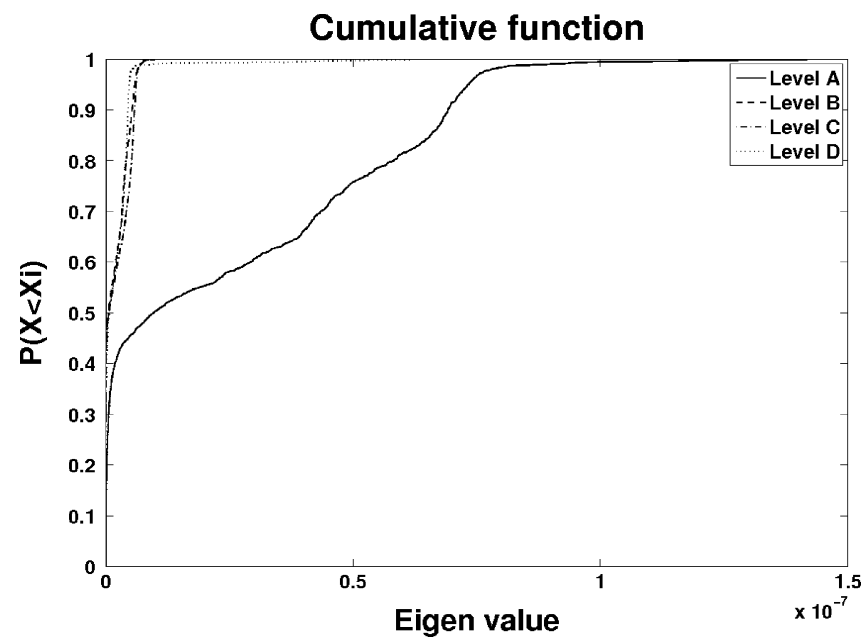

Fig. 18. Entrance hall environment: cumulative functions associated with the eigenvalues in NLOS configuration, without polarization diversity.

\section{B. Summary}

Before turning attention to MIMO characterization in relation to the number of antennas, the above results are summarized. It has been shown that channel characteristic parameters are not sensitive to electrical description, whatever the configuration of the environment and whatever the polarization.

Furthermore, it is asserted that geometrical description has an impact on MIMO characterization. This fact is amplified in the NLOS configuration because of the lack of a direct path. At larger-scale description, it is shown that architectural elements outside the rooms involved in wireless transmission have little influence on MIMO channel characterization. Accordingly, level B would seem to be a satisfactory level of description for accomplishing an adequate characterization of a MIMO channel. This allows a gain in computation time over level D roughly to the order of two.

\section{Variation of Characteristic Parameters in Relation to the Number of Antennas}

To build on the above findings, the following results evaluate gains in capacity in relation to the number of antennas (cf. Section IV.B), for LOS and NLOS cases, with and without polarization diversity. The aim of this part is to examine previous assumptions for more than two transmitter and receiver antennas.

1) LOS Configuration Without Polarization Diversity: Figs. 19 and 20 show an increasing gain in capacity in relation to the number of antennas. This is line with the study of Yun and Iskander [5]. Moreover, these figures clearly show that a minimum level of modeling is necessary as the number of antennas increases. In fact, where there are more than two transmitter and receiver antennas, the simplest modeling (level A) is not sufficient to adequately evaluate MIMO channel characteristic parameters. It thus appears that a geometric level of description is of major importance for MIMO characterization. A minimum level of description is required to take into account the propagation of predominant paths. In spite of this, it is not necessary to consider all geometric elements (i.e., all rooms). In the end, an electrical description of materials has less impact on MIMO channel characterization than a geometrical description.

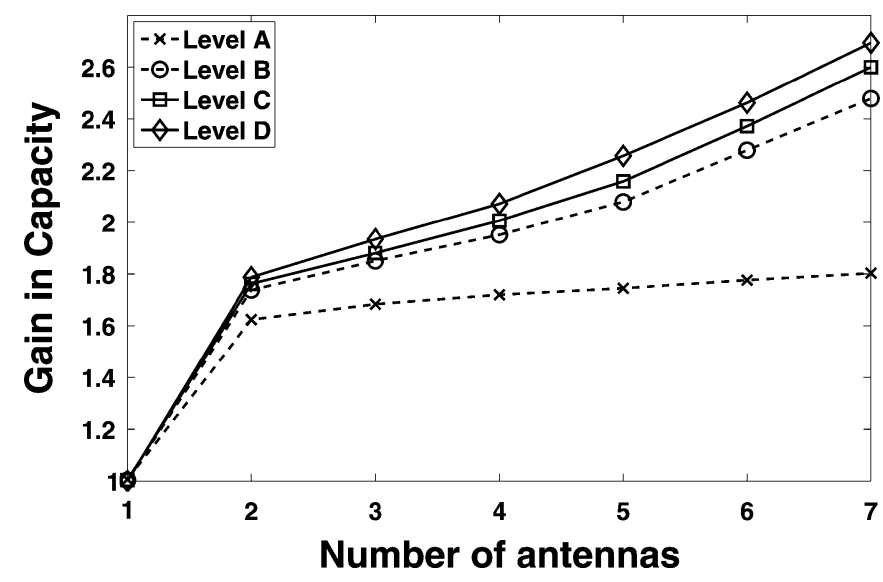

Fig. 19. Entrance hall environment: variation in the gain in capacity in relation to the number of antennas in a LOS configuration, without polarization diversity. (The element spacing is equal to $0.4 \lambda$ ).

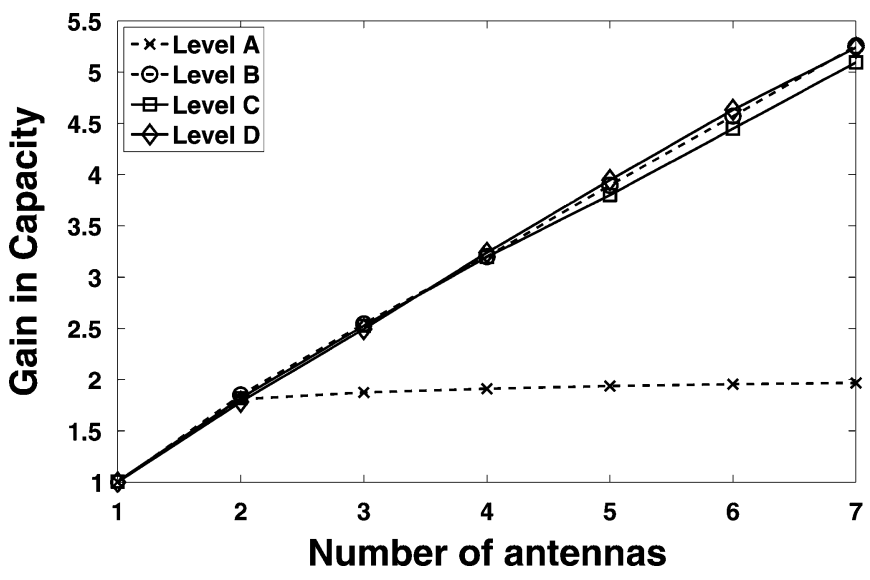

Fig. 20. First floor environment: variation in the gain in capacity in relation to the number of antennas in a LOS configuration, without polarization diversity. (The element spacing is equal to $0.4 \lambda$ ).

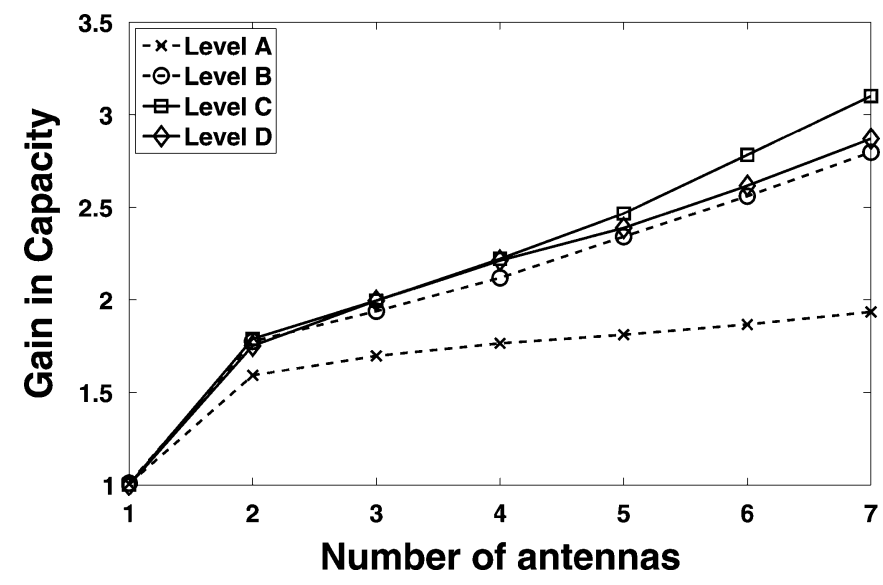

Fig. 21. Entrance hall environment: variation in the gain in capacity in relation to the number of antennas in a LOS configuration, with polarization diversity. (The element spacing is equal to $0.4 \lambda$ ).

2) LOS Configuration With Polarization Diversity: Polarization diversity brings about the same gain in capacity as in the previous configuration (compare Figs. 21 and 22 and Figs. 19 and 20). Thus, these results corroborate the analysis of the previous section. 


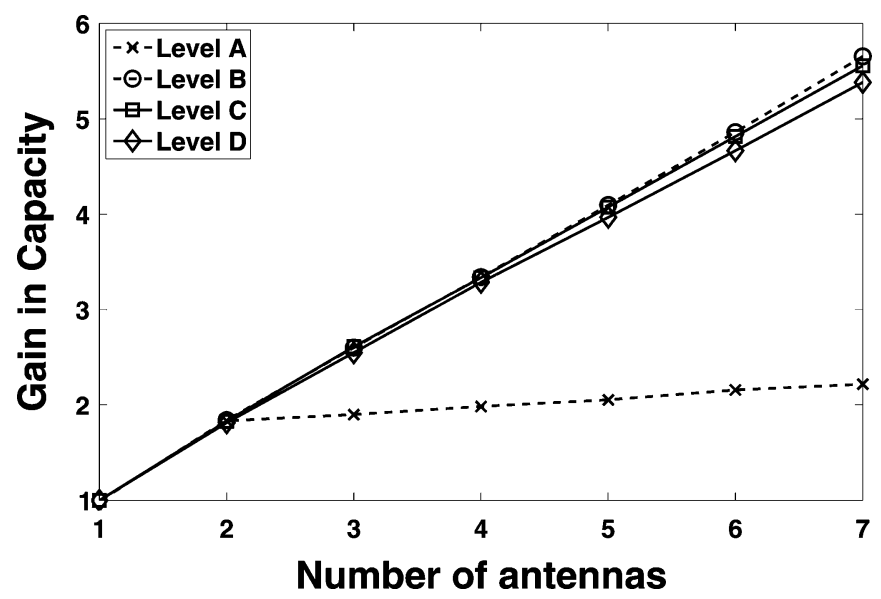

Fig. 22. First floor environment: variation in the gain in capacity in relation to the number of antennas in a LOS configuration, with polarization diversity. (The element spacing is equal to $0.4 \lambda$ ).

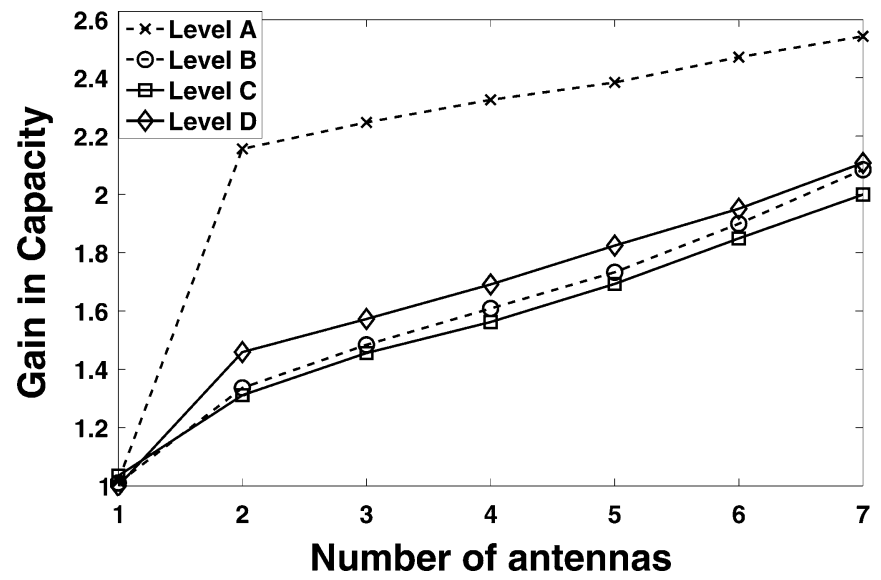

Fig. 23. Entrance hall environment: variation in the gain in capacity in relation to the number of antennas in a NLOS configuration, without polarization diversity. (The element spacing is equal to $0.4 \lambda$ ).

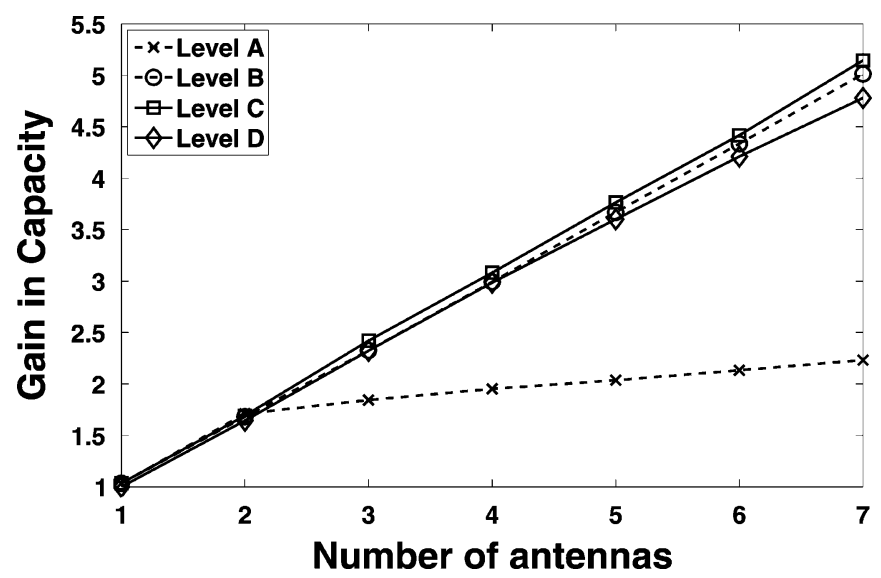

Fig. 24. First floor environment: variation in the gain in capacity in relation to the number of antennas in a NLOS configuration, without polarization diversity. (The element spacing is equal to $0.4 \lambda$ ).

3) NLOS Configuration Without Polarization Diversity: The study of the lack of a direct path in the Section V.A.3 has shown that the influence of environment modeling emerges in this condition. The contrast of these results (see Figs. 23 and 24) and those presented in Section V.A.3 supports this assumption.

\section{CONCLUSION}

This work has investigated environment modeling effects on MIMO channel characterization. The goal was to answer to the question: What degree of accuracy is required to describe the indoor environment, both geometrically and electrically, to arrive at an adequately accurate MIMO channel characterization involving a minimum of computation time?

To highlight modeling effects, correlation matrices and gain in capacity values, multipath richness and eigenvalues were used. These characteristic parameters were investigated in relation to the spacing between antennas on the one hand, and the number of antennas on the other hand. Moreover, environment modeling effects were studied in LOS and NLOS cases, with or without polarization diversity. All the cases studied revealed that MIMO characterization is quite insensitive to the electrical properties of materials. In the same context, the results show that rooms beside those directly concerned by the transmission have a little impact on MIMO channel characterization. However, geometrical precision in the description of rooms is a crucial parameter in achieving an accurate characterization. Indeed, characteristic parameters vary strongly where modeling of the environment involves poor geometric description. This fact is amplified in NLOS configurations where a direct path is lacking. It is concluded that pertinent environment modeling requires a minimum level of description of geometric elements involved in propagation configurations. Further, materials can be described uniformly as having the electrical properties of concrete. It is consequently assumed that level B ensures an adequate level of descriptive accuracy, while offering a gain in computation time roughly of the order of two, when compared with level D descriptions.

\section{REFERENCES}

[1] J. Kivinen, Z. Xiongwen, and P. Vainikainen, "Empirical characterization of wideband indoor radio channel at $5.3 \mathrm{ghz}$," IEEE Trans. Antennas Propag., vol. 49, no. 8, pp. 1192-1203, Aug. 2001.

[2] A. Abdi and M. Kaveh, "A space-time correlation model for multielement antenna systems in mobile fading channels," IEEE J. Sel. Areas. Commun., vol. 20, no. 3, pp. 550-560, Apr. 2002.

[3] J. W. Wallace, M. A. Jensen, A. L. Swindlehurst, and B. D. Jeffs, "Experimental characterization of the MIMO wireless channel: Data acquisition and analysis," IEEE Trans. Wireless Commun., vol. 2, no. 2, pp. 335-343, Mar. 2003.

[4] P. Kyritsi, D. C. Cox, R. A. Valenzuela, and P. W. Wolniansky, "Effect of antenna polarization on the capacity of a multiple element system in an indoor environment," IEEE J. Sel. Areas. Commun., vol. 20, no. 6, pp. 1227-1239, Aug. 2002.

[5] Z. Yun, M. F. Iskander, and Z. Zhang, "Complex-wall effect on propagation characteristics and MIMO capacities for an indoor wireless communication environment," IEEE Trans. Antennas Propag., vol. 52, no. 4, pp. 914-922, Apr. 2004.

[6] D. W. Browne, J. Medbo, H. Asplund, and J. E. Berg, "A simple approach to site sensitive modeling of indoor radio propagation," in Proc. IEEE 55th Vehic. Technol. Conf., 2002, vol. 1, pp. 384-388.

[7] F. Mora and L. Aveneau, "Optimised scanning of a visibility graph data structure for efficient ray-tracing," in Proc. Eur. Conf. on Wireless Technol., Oct. 2005, vol. 1, pp. 35-38.

[8] F. Escarieu, Y. Pousset, L. Aveneau, and R. Vauzelle, "Outdoor and indoor channel characterization by a 3D simulation software," in Proc. IEEE Int. Symp. on Personal Indoor and Mobile Radio Commun., Sep. 2001, vol. 1, pp. 105-111.

[9] Y. Chartois, Y. Pousset, and R. Vauzelle, "A SISO and MIMO radio channel characterization with a $3 \mathrm{D}$ ray tracing propagation model in urban environment," presented at the Eur. Conf. on Propag. and Syst., Mar. 2005. 
[10] M. Steinbauer, A. F. Molisch, and E. Bonek, "The double-directional radio channel," IEEE Antennas Propag. Mag., vol. 43, no. 4, pp. 51-63, Apr. 2001.

[11] J. L. Rodgers and W. A. Nicewander, "Thirteen ways to look at the correlation coefficient," Amer. Statistician, vol. 42, no. 1, pp. 59-66, Feb. 1988.

[12] C. E. Shannon, A Mathematical Theory of Communication Oct. 1948.

[13] D. Gesbert and J. Akhtar, "Breaking the barriers of Shannon's capacity: An overview of MIMO wireless system," Telektronikk Telenor J., Jan. 2002.

[14] R. Vauzelle, Y. Pousset, and F. Escarieu, "A sensitivity study for an indoor channel," Annals of Telecommun., vol. 59, no. 5-6, pp. 713-730, Feb. 2004

[15] F. Escarieu, "Etude de la propagation dans les milieux indoor autour de 2 GHz.," Ph.D. dissertation, Univ. Poitiers, Poitiers, France, Dec. 2002.

[16] C. N. Chuah, D. N. C. Tse, J. M. Kahn, and R. A. Valenzuela, "Capacity scaling in MIMO wireless systems under correlated fading," IEEE Tran. Inf. Theory, vol. 48, no. 3, pp. 637-650, Mar. 2002.

[17] Z. Tang and A. S. Mohan, "Indoor multipath characterization for MIMO wireless communications," in Auswireless Conf., Mar. 13-16, 2006.

[18] J. W. Wallace and M. A. Jensen, "MIMO capacity variation with SNR and multipath richness from full-wave indoor FDTD simulations," in Proc. IEEE Antennas and Propag. Society Int. Symp., Jun. 2003, vol. 2, no. 22-27, pp. 523-526.

[19] T. Svantesson and J. W. Wallace, "On signal strength and multipath richness in multi-input multi-output systems," in IEEE Int. Conf. on Commun., May 2003, vol. 4, no. 11-15, pp. 2683-2687.

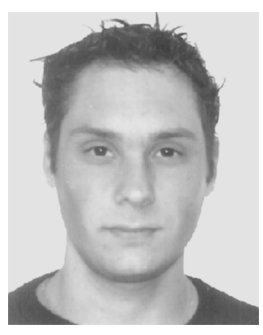

tween antennas.
Pereira Carlos (S'05) received the M.S. degree in mobile radiocommunication from the University of Poitiers, France, in 2005, where he is currently working toward the Ph.D. degree.

Currently, he works as a Ph.D. student in mobile radiocommunication in a collaboration context between the Signal Image and Communication (SIC) Laboratory at the University of Poitiers and the Department of Micro-Wave at ENST-Bretagne, Brest, France. His research interests include MIMO channel characterization and Mutual coupling be-

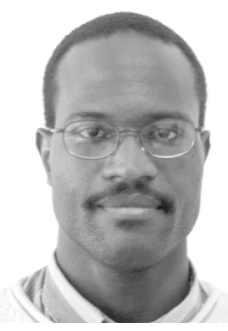

Pousset Yannis received the Ph.D. degree in mobile radiocommunication from the University of Poitiers, Poitiers, France, in 1998.

Since 2000, he has been an Assistant Professor in the Department of Electrical Engineering, University of Poitiers, where he also develops research activities in the Signal Image and Communication Laboratory. His research interests include the study of the electromagnetic waves propagation for the SISO and MIMO wireless communication systems.

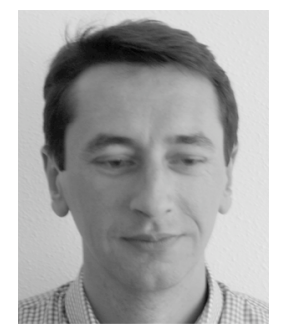

Vauzelle Rodolphe received the Ph.D. degree in mobile radiocommunication from the University of Poitiers, Poitiers, France, in 1994.

From 1994 to 2004, he was an Assistant Professor in the Electrical Department, University of Poitiers, where, since 2005, he has been a Professor. He also develops research activities in the Signal Image and Communication Laboratory at the same university. His research interests focus the realistic simulation of the physical layer of ad'hoc network based on SISO and MIMO channel modeling.

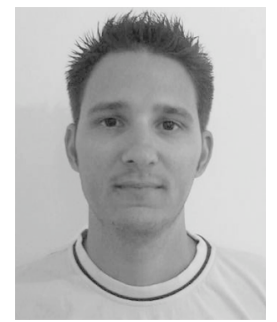

Combeau Pierre received M.S. degree in mobile radiocommunication and image processing and the $\mathrm{Ph} . \mathrm{D}$. degree in signal processing and telecommunications from the University of Poitiers, Poitiers, France, in 2001 and 2004, respecitvely.

Since 2005, he has been an Assistant Professor in the Department of Electrical Engineering, University of Poitiers. His research interests include the study of the electromagnetic waves propagation for the SISO and MIMO wireless communication systems. 